\title{
Electro-mechanical coupling of rotating 3D beams
}

\author{
S. Stoykov ${ }^{1}$ and E. Manoach ${ }^{2}$ \\ ${ }^{1}$ Institute of Information and Communication Technologies, Bulgarian Academy of Sciences, Bulgaria \\ ${ }^{2}$ Institute of Mechanics, Bulgarian Academy of Sciences, Bulgaria
}

\begin{abstract}
A rotating thin-walled beam with piezoelectric element is analysed. The beam is considered to vibrate in space, hence the longitudinal, transverse and torsional deformations are taken into account. The bending deformations of the beam are modelled by assuming Timoshenko's theory. Torsion is included by considering that the cross section rotates as a rigid body but can deform in longitudinal direction due to warping. The warping function is computed preliminary by the finite element method. The equation of motion is derived by the principle of virtual work and discretized in space by the Ritz method. Electro-mechanical coupling is included in the model by considering the internal electrical energy and the electric charge output. The piezo-electric constitutive relations are used in reduced form. The beam is assumed to rotate about a fixed axis with constant speed. The equation of motion is derived in rotating coordinate system, but the influence of the rotation of the coordinate system is taken into account through the inertia forces. Results in time domain are presented for different speeds of rotation and frequencies of vibration. The influence of the speed of rotation and of the frequency of vibration on the electrical output is presented and analysed.
\end{abstract}

\section{Introduction}

In the last decades, the need of independent energy sources for small devices has increased significantly. Small devices, such as sensors, have application in health monitoring and control of structures. The most popular devices that convert kinetic energy into electrical energy are the devices composed of piezoelectric elements [1].

In the current work, a rotating thin-walled beam with piezoelectric element is analysed. Three-dimensional displacement field is considered, i.e. the beam can vibrate in both transverse directions and it can perform longitudinal and torsional deformations. The beam equation of motion is derived by Timoshenko's beam assumption and it is considered that under torsion the cross section rotates as a rigid body but it can deform in longitudinal direction due to warping. The proposed model is suitable for beams with arbitrary open and closed thin-walled cross sections as well for solid cross sections. Clamped-free boundary conditions are assumed and rotation of the beam about fixed axis with constant speed is included in the model. The piezoelectric element is considered to be close to the clamped end. Geometrical nonlinearity is considered and electro-mechanical coupling is introduced into the model through the piezoelectric constitutive equations, used in reduced form.

The equation of motion is derived by the principle of virtual work and results in time domain are presented. Comparison between linear and nonlinear models is shown and the importance of the geometrical nonlinear terms on the displacements and on the electrical potential difference is outlined. The responses of the beam due to harmonic force and different speeds of rotation are compared.

\section{Mathematical model}

A thin-walled beam with piezoelectric element on the upper surface is considered (Fig. 1). The piezoelectric element does not cover the whole length of the beam. The displacements in longitudinal and transverse directions are expressed by the displacements and rotations on the reference line:

$$
\begin{aligned}
& u(x, y, z, t)=u_{0}-y \phi_{z}+z \phi_{y}+\psi \frac{\partial \theta_{x}}{\partial x} \\
& v(x, y, z, t)=v_{0}+y \cos \left(\theta_{x}\right)-y-z \sin \left(\theta_{x}\right) \\
& w(x, y, z, t)=w_{0}+y \sin \left(\theta_{x}\right)+z \cos \left(\theta_{x}\right)-z
\end{aligned}
$$

where $u_{0}(x, t), v_{0}(x, t)$ and $w_{0}(x, t)$ represent the displacements in longitudinal and transverse displacements in $y$ and $z$ axes, respectively. $\phi_{y}(x, t)$ and $\phi_{z}(x, t)$ represent the rotations of the cross section about $y$ and $z$ axes, $\theta_{x}(x, t)$ denotes the torsion and $\psi(y, z)$ is the warping function. 
Fig. 1. Thin-walled beam with piezoelectric element.

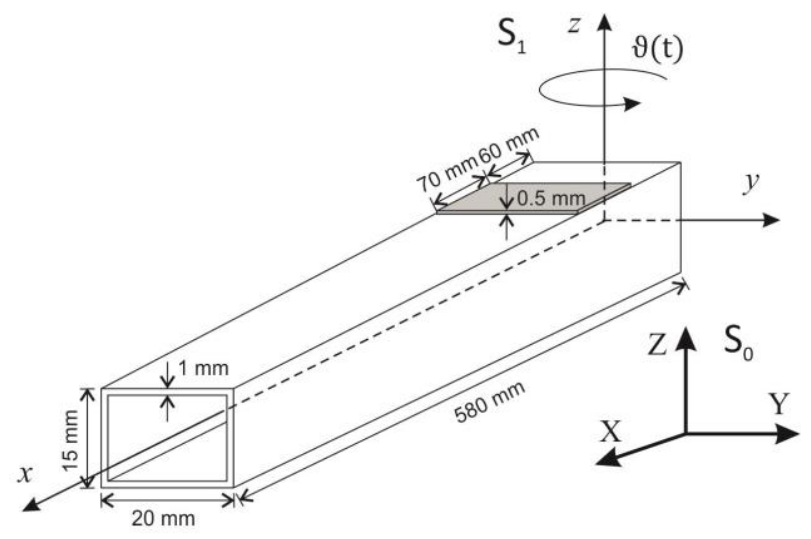

Analytical solution for the warping function does not exist for complex cross sections, thus it is computed numerically. The equation of equilibrium for the warping function is derived from the equilibrium equations of elasticity, where the stresses due to torsion are computed from the displacement field:

$\tilde{C}_{66}^{k} \frac{\partial^{2} \psi}{\partial y^{2}}+\tilde{C}_{55}^{k} \frac{\partial^{2} \psi}{\partial z^{2}}=0$ in $\Omega_{k}$

$\left(\tilde{C}_{66}^{k}-\tilde{C}_{66}^{k-1}\right)\left(\frac{\partial \psi}{\partial y}-z\right) n_{y}+$

$+\left(\tilde{C}_{55}^{k}-\tilde{C}_{55}^{k-1}\right)\left(\frac{\partial \psi}{\partial z}+y\right) n_{z}=0 \quad$ on $\Gamma_{k}$

The equation for the warping function is given for the case of beam with composite materials, because of the piezoelectric element. For the current beam, two warping functions are computed - one for the cross section without piezoelectric element and another one for the cross section with the piezoelectric element. In eq. (2) $\Omega_{k}$ is the area of the $k^{\text {th }}$ material and $\Gamma_{k}$ is its contour. $\tilde{C}_{55}^{k}$ and $\tilde{C}_{66}^{k}$ are the shear moduli of the material from the reduced stiffness matrix. $n_{y}$ and $n_{z}$ are the components of the normal vector to the boundary. The normal vector points in the outer direction for the outer boundaries and it is considered to point in the lower numbered material for the boundaries between different layers. $\tilde{C}_{66}^{k-1}=$ $\tilde{C}_{55}^{k-1}=0$ for the outer boundaries. Equation (2) is solved by the finite element method [2].

Geometrical nonlinearity is considered in the model. Hence the strains are expressed as:

$\varepsilon_{x}=\frac{\partial u}{\partial x}+\frac{1}{2}\left(\frac{\partial v}{\partial x}\right)^{2}+\frac{1}{2}\left(\frac{\partial w}{\partial x}\right)^{2}$

$\gamma_{x z}=\frac{\partial w}{\partial x}+\frac{\partial u}{\partial z}+\frac{\partial v}{\partial z} \frac{\partial v}{\partial x}+\frac{\partial w}{\partial z} \frac{\partial w}{\partial x}$

$\gamma_{x y}=\frac{\partial u}{\partial y}+\frac{\partial v}{\partial x}+\frac{\partial v}{\partial x} \frac{\partial v}{\partial y}+\frac{\partial w}{\partial x} \frac{\partial w}{\partial y}$

The piezo-electric constitutive equations are used in their reduced form [1]:

$$
\left\{\begin{array}{c}
\sigma_{x} \\
\tau_{x z} \\
\tau_{x y} \\
D_{3}
\end{array}\right\}=\left[\begin{array}{cccc}
E & 0 & 0 & -\bar{e}_{31} \\
0 & G & 0 & 0 \\
0 & 0 & G & 0 \\
\bar{e}_{31} & 0 & 0 & \bar{\varepsilon}_{33}^{S}
\end{array}\right]\left\{\begin{array}{c}
\varepsilon_{x} \\
\gamma_{x z} \\
\gamma_{x y} \\
E_{3}
\end{array}\right\}
$$

where $\sigma_{x}, \tau_{x z}$ and $\tau_{x z}$ are bending and shear stresses, $E$ is the Young's modulus of the material, $G$ is the shear modulus, $D_{3}$ represents electric displacement component, $\bar{e}_{31}$ is an effective piezo-electric stress constant, $\bar{\varepsilon}_{33}^{S}$ is permittivity component and $E_{3}$ is electric field component. The electric field component can be presented as $E_{3}=-\frac{v(t)}{h_{p}}$ where $v(t)$ is an electric potential difference (voltage) and $h_{p}$ is the thickness of the piezoelectric element.

The rotation of the beam is taken into account by considering two coordinate systems: one fixed, denoted by $S_{0}$, and another one rotating about the fixed coordinate system denoted by $S_{1}$. The displacement fields (1) are written in the rotating coordinate system $S_{1}$, it is considered that it rotates with constant speed $\dot{\vartheta}(\mathrm{rad} / \mathrm{s})$. The rotation of the beam is introduced into the equation of motion through the inertia forces. For that purpose, the absolute acceleration of an arbitrary point $\mathrm{P}(x, y, z)$ from the beam is expressed with respect to the fixed coordinate system $S_{0}[3]$ :

$a_{\mathrm{P}_{\mathrm{S}_{0}}}=a_{\mathrm{P}_{\mathrm{S}_{1}}}+\boldsymbol{\omega} \times(\boldsymbol{\omega} \times \boldsymbol{r})+2 \boldsymbol{\omega} \times \boldsymbol{v}_{\mathrm{P}_{\mathrm{S}_{1}}}$

where $\boldsymbol{a}_{\mathrm{P}_{\mathrm{s}_{0}}}$ is the absolute acceleration of point $\mathrm{P}$, i.e. the acceleration of point $\mathrm{P}$ with respect to $S_{0}, \boldsymbol{a}_{\mathrm{P}_{\mathrm{S}_{1}}}$ is the relative acceleration of point $\mathrm{P}$, i.e. the acceleration of point $\mathrm{P}$ with respect to $S_{1}, \boldsymbol{v}_{\mathrm{P}_{\mathrm{S}_{1}}}$ is the relative velocity of point $\mathrm{P}, \omega$ is the angular velocity vector of the rotating coordinate system, and $\boldsymbol{r}$ is the position vector of point $\mathrm{P}$ with respect to the origin of $S_{1}$. In eq. (4) $2 \omega_{10} \times v_{\mathrm{P}_{\mathrm{S}_{1}}}$ represents the acceleration of Coriolis.

The equation of motion is derived by the principle of virtual work:

$\delta W_{V}+\delta W_{i n}+\delta W_{E}+\delta W_{i e}+\delta W_{n c}=0$

where $\delta W_{V}$ is the virtual work of internal forces, $\delta W_{i n}$ is the virtual work of inertia forces, $\delta W_{E}$ is the virtual work of external forces, $\delta W_{i e}$ is the virtual work of internal electrical energy and $\delta W_{n c}$ is the non-conservative virtual work due to electric charge output.

The displacement components are expressed by shape functions and generalized coordinates. The shape functions used in [2] are used also here. They have to satisfy the essential boundary conditions. Applying the principle of virtual work, the following system of ordinary differential equations is obtained:

$$
\begin{aligned}
& \mathbf{M} \ddot{\mathbf{q}}+\beta \mathbf{K}_{\mathbf{l}} \dot{\mathbf{q}}+\mathbf{C R}(\mathbf{q}, \dot{\vartheta}) \dot{\mathbf{q}}+\mathbf{K}_{\mathbf{l}} \mathbf{q}+\mathbf{K}_{\mathbf{n l}}(\mathbf{q}) \mathbf{q}+ \\
& +\mathbf{T}(\mathbf{q}, \dot{\vartheta}) \mathbf{q}+\boldsymbol{\theta}_{\mathbf{l}} v+\boldsymbol{\theta}_{\mathbf{n l}}(\mathbf{q}) v=\mathbf{f}-\mathbf{r}(\dot{\vartheta}) \\
& C_{p} v+Q-\boldsymbol{\theta}_{\mathbf{l}}^{\mathrm{T}} \mathbf{q}-\frac{1}{2} \boldsymbol{\theta}_{\mathbf{n l}}^{\mathrm{T}}(\mathbf{q}) \mathbf{q}=0
\end{aligned}
$$

where $\mathbf{M}$ is the mass matrix, $\mathbf{K}_{\mathbf{l}}$ is the stiffness matrix of constant terms, $\mathbf{K}_{\mathbf{n l}}(\mathbf{q})$ is the stiffness matrix that depends on the vector of generalized coordinates $\mathbf{q}$. $\mathbf{K}_{\mathbf{n l}}(\mathbf{q})$ results from the geometrical nonlinearity of the model and introduces quadratic and cubic terms at the equation of motion, $\boldsymbol{\theta}_{\mathbf{l}}$ is vector of constant terms which 
results from the electro-mechanical coupling, $\boldsymbol{\theta}_{\mathbf{n l}}(\mathbf{q})$ is vector which also results from the electro-mechanical coupling but it depends linearly on the vector of generalized coordinates $\mathbf{q}$. $f$ represents the vector of generalised external forces, $Q$ represents the electric charge output on the electrodes and $C_{p}$ is the corresponding capacitance. $\mathbf{C R}(\dot{\vartheta})$ is matrix that depends on the speed of rotation, it results from the acceleration of Coriolis and $\mathbf{T}(\dot{\vartheta})$ and $\mathbf{r}(\dot{\vartheta})$ are matrix and vector that also arise from the rotation of the beam and depend on the speed of rotation. Stiffness proportional damping is introduced in the model. The equation of motion (6) is solved in time domain by the Newmark's method.

\section{Numerical results}

\subsection{Validation}

The derived equation of motion is validated by comparing the linear natural frequencies with results from the three-dimensional equation of elasticity applied to the same structure. The software Elmer [4] is used for that purpose and space discretization is achieved by quadratic tetrahedrons. The beam is considered to be composed of brass and the piezoelectric element is considered to be PZT-5A [1]. The material properties are given in Table 1.

Table 1. Material properties of the structure.

\begin{tabular}{|c|c|c|}
\hline & Brass & PZT-5A \\
\hline Young modulus $(E)$ & $105 \mathrm{GPa}$ & $60.6 \mathrm{GPa}$ \\
\hline Shear modulus $(G)$ & $40 \mathrm{GPa}$ & $23 \mathrm{GPa}$ \\
\hline Density $(\rho)$ & $9000 \mathrm{~kg} / \mathrm{m}^{3}$ & $7500 \mathrm{~kg} / \mathrm{m}^{3}$ \\
\hline $\begin{array}{c}\text { Piezo-electric stress } \\
\text { constant }\left(\bar{e}_{31}\right)\end{array}$ & - & $-10.4 \mathrm{C} / \mathrm{m}^{2}$ \\
\hline Permittivity constant $\left(\bar{\varepsilon}_{33}^{\mathrm{S}}\right)$ & - & $13.3 \times 10^{-9} \mathrm{~F} / \mathrm{m}$ \\
\hline
\end{tabular}

In the comparison of the natural frequencies, only the elastic properties of the piezoelectric element are taken into account. Clamped-free boundary conditions are considered. The first six natural frequencies are presented in Table 2. The results confirm that very good approximation is obtained by the proposed beam model.

Table 2. Validation of natural frequencies $(\mathrm{rad} / \mathrm{s})$ of the beam with piezoelectric element.

\begin{tabular}{|c|c|c|c|}
\hline Mode & $\begin{array}{c}\text { Elmer 3D } \\
300000 \text { DOF }\end{array}$ & $\begin{array}{c}\text { Beam model } \\
60 \text { DOF }\end{array}$ & $\begin{array}{c}\text { Difference } \\
\%\end{array}$ \\
\hline 1 & 215.5521 & 215.8158 & 0.12 \\
\hline 2 & 268.1170 & 268.1142 & 0.01 \\
\hline 3 & 1312.9806 & 1321.1389 & 0.62 \\
\hline 4 & 1646.1054 & 1651.9888 & 0.36 \\
\hline 5 & 3587.8169 & 3645.1821 & 1.60 \\
\hline 6 & 4490.5568 & 4533.8394 & 0.96 \\
\hline
\end{tabular}

\subsection{Influence of nonlinear terms}

A comparison of the response of the beam between the linear and the geometrically nonlinear models is presented in this sub-section. A transverse force in $z$ direction is applied on the free end of the beam and the rotation is not considered. The transverse displacement $w_{0}$ is shown in Fig. 2 and the electric potential difference, which results from the deformation of the beam due to the external force, is presented in Fig. 3. The excitation frequency is chosen to be one third from the fundamental frequency. The difference between the linear and nonlinear models is obvious not only on the displacements, but also on the electrical potential difference, which confirms that geometrical nonlinear terms are essential for accurate results.

Fig. 2. Transverse displacement $w_{0}$, at the free end, due to harmonic force $F_{z}=100 \cos (71.94 t)$ applied at the free end in $z$ direction, $\beta=10^{-3}$. - nonlinear model,--- - linear model.

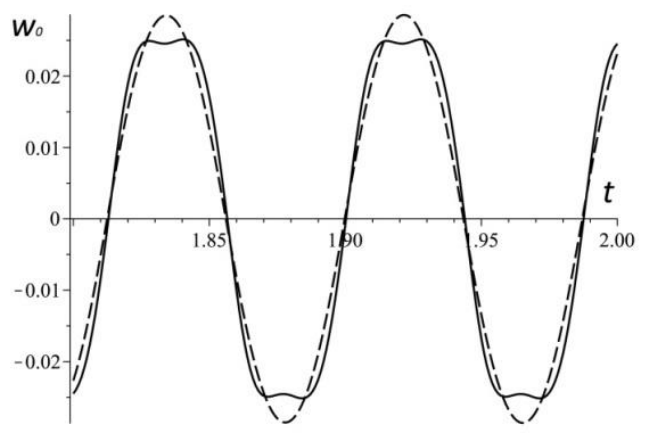

Fig. 3. Electric potential difference $v(t)$ due to harmonic force $F_{z}=100 \cos (71.94 t),-$ nonlinear , --- linear model.

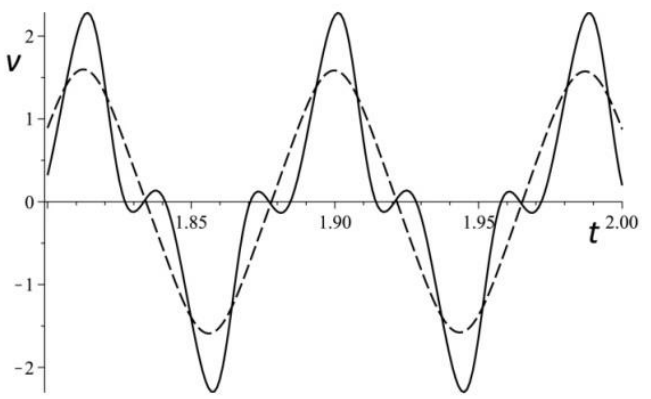

\subsection{Influence of speed of rotation}

The influence of the speed of rotation on the displacements and on the electrical potential difference is analysed in this sub-section. It is known that the speed of rotation makes the beam stiffer, due to the centrifugal forces, and the natural frequencies become higher. The first four natural frequencies for different speeds of rotation are presented in Table 3.

Table 3. Natural frequencies ( $\mathrm{rad} / \mathrm{s})$ of rotating beam with different speeds of rotation $(\mathrm{rad} / \mathrm{s})$

\begin{tabular}{|c|c|c|c|c|}
\hline ModelSpeed & 0 & 50 & 146 & 250 \\
\hline 1 & 215.82 & 222.61 & 268.07 & 288.42 \\
\hline 2 & 268.11 & 269.01 & 275.48 & 346.47 \\
\hline 3 & 1321.14 & 1327.20 & 1371.95 & 1465.22 \\
\hline 4 & 1651.99 & 1656.08 & 1686.55 & 1751.46 \\
\hline
\end{tabular}


In order to excite both transverse displacements and the torsion and to have vibration in the three-dimensional space, harmonic forces are applied on the free end of the beam in directions of both transverse axes. An excitation frequency equal to the second natural frequency of the beam, when it does not rotate, is considered. This frequency is related with the first mode of vibration in $x y$ plane, while the fundamental frequency is related with first mode in $x z$ plane. The excitation frequency becomes fundamental frequency of the beam when the beam rotates with speed of $146 \mathrm{rad} / \mathrm{s}$.

Fig. 4. Electric potential difference $v(t)$ due to external force $F_{y}=F_{z}=100 \cos (268 t)$ and non-rotating beam.

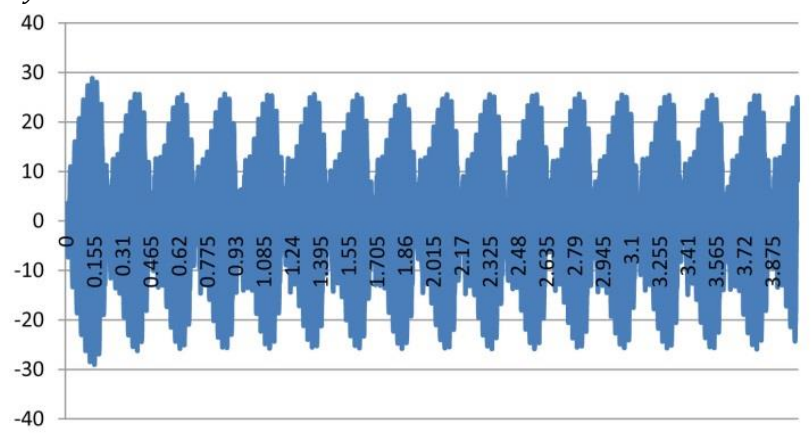

The resulting electric potential difference is presented in figures 4 to 7, for the cases of non-rotating beam, and for rotating beam with speeds of rotation respectively 50 $\mathrm{rad} / \mathrm{s}, 146 \mathrm{rad} / \mathrm{s}$ and $250 \mathrm{rad} / \mathrm{s}$.

Fig. 5. Electric potential difference $v(t)$ due to external force $F_{y}=F_{z}=100 \cos (268 t)$ and rotation with speed $50 \mathrm{rad} / \mathrm{s}$.

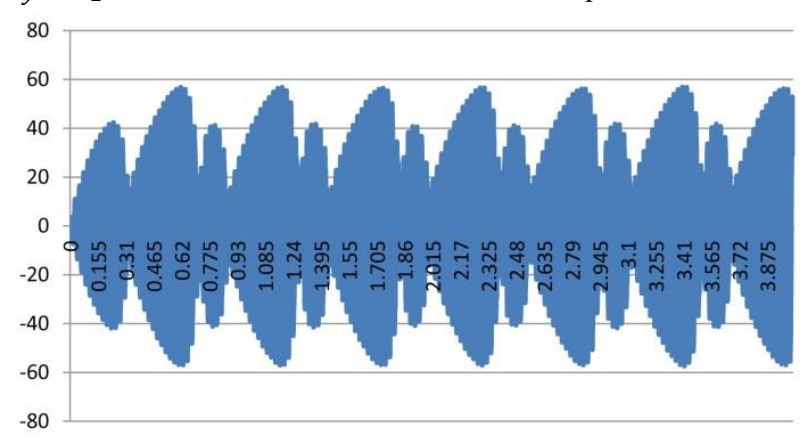

The results demonstrate that the speed of rotation influences significantly on the electrical potential difference and depending on the speed of rotation, different amounts of energy are generated.

Fig. 6. Electric potential difference $v(t)$ due to external force $F_{y}=F_{z}=100 \cos (268 t)$ and rotation with speed $146 \mathrm{rad} / \mathrm{s}$.

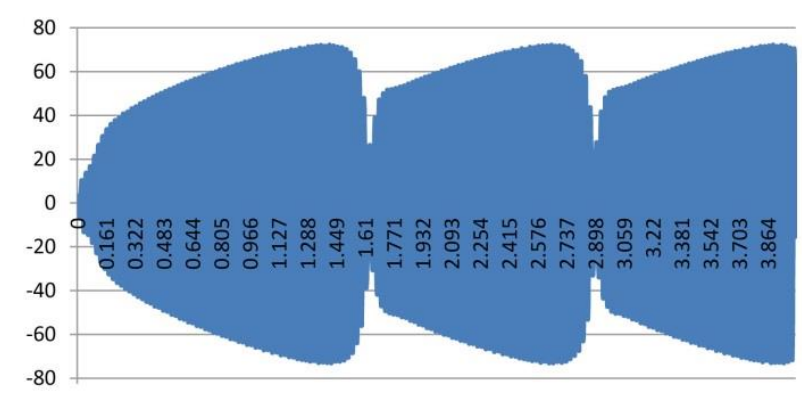

Fig.7. Electric potential difference $v(t)$ due to external force $F_{y}=F_{z}=100 \cos (268 t)$ and rotation with speed $200 \mathrm{rad} / \mathrm{s}$.

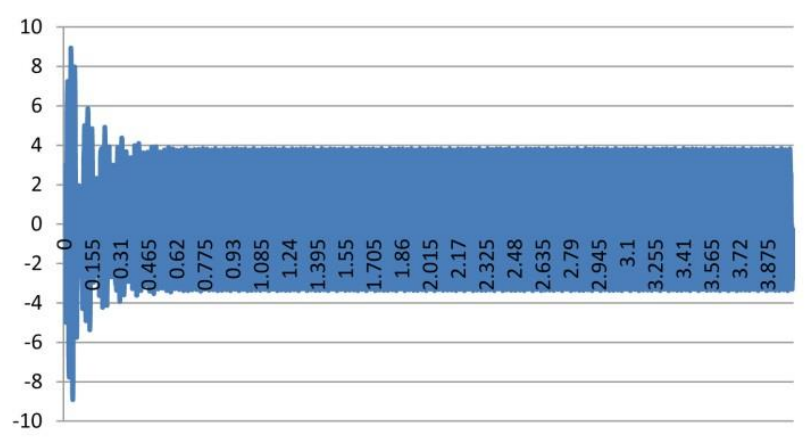

For the cases of non-rotating beam and beam rotating with speeds of $50 \mathrm{rad} / \mathrm{s}$ and $146 \mathrm{rad} / \mathrm{s}$ the excitation frequency of the external force is between first two natural frequencies. Because of the nonlinear terms and the interaction between first two modes the response of the beam becomes complex. Consequently the electrical potential difference is also complex. For the case of beam rotating with speed of $250 \mathrm{rad} / \mathrm{s}$, the excitation frequency is smaller than the fundamental one, and the response of the beam is similar to harmonic with small amplitude. Thus, the resulting potential difference is also similar to harmonic and the amplitude is smaller than in the previous cases.

\section{Conclusion}

The equation of motion of rotating beam with piezoelectric element is derived considering geometrical type of nonlinearity and introducing electro-mechanical coupling. The importance of the nonlinear terms on the response of the beam is presented. The influence of the speed of rotation on the electric potential difference is outlined and it is shown that the speed of rotation can change significantly the response of the beam and the resulting electrical output.

\section{Acknowledgements}

The support of this work through the Bulgarian NSF Grant DUNK-01/3 is gratefully acknowledged. The second author acknowledges the support through H2020 Marie Sklodowska-Curie ITN grant (grant agreement No 643095).

\section{References}

1. A. Erturk, D. Inman, Piezoelectric energy harvesting, (John Wiley \& Sons Ltd. 2011)

2. S. Stoykov, E. Manoach, S. Margenov, An efficient 3D numerical beam model based on cross sectional analysis and Ritz approximations, Z. Angew. Math. Mech. (2015), DOI 10.1002/zamm.201400139 (In Press)

3. A. Shabana, Dynamics of multibody systems, (Cambridge University Press, 2013)

4. Elmer web site: www.csc.fi/elmer (last time visited 02.06.2016) 